\title{
Influence of the filler on thermal properties of porous VP-TRIM copolymers
}

\author{
M. Maciejewska
}

Received: 8 April 2014/ Accepted: 19 August 2014/Published online: 12 September 2014

(C) The Author(s) 2014. This article is published with open access at Springerlink.com

\begin{abstract}
In this paper, the synthesis and characterization of thermal properties of porous copolymers 1-vinyl-2pyrrolidone with trimethylolpropane trimethacrylate are presented. They were obtained by suspension polymerization as a pure polymers or composite materials with different inorganic fillers. The influence of the type of filler on the textural and thermal properties was investigated in details. It was found that the value of the porous surface area of composites is much lower than in the case of pure copolymers. Thermal properties of the obtained materials were investigated by the means of thermogravimetry and differential scanning calorimetry.
\end{abstract}

Keywords Thermal properties - Thermogravimetry · Differential scanning calorimetry $\cdot$ Fillers · Porous copolymers $\cdot$ 1-vinyl-2-pyrrolidone

\section{Introduction}

One of the dominant trends in science and technology is searching for new efficient adsorbents. In huge variety of different types of materials, polymers offer unlimited possibilities to create new materials that have the ability to adsorb the required substance. Beside synthesis of new types polymers, copolymers, terpolymers or semi-interpenetrating polymer network [1-14] modification of polymeric matrix with different fillers is widely applied [15-23]. Quite a large variety of inorganic particles can be incorporated into polymer matrix. Recently, we have

M. Maciejewska ( $\square)$

Faculty of Chemistry, Maria Curie-Skłodowska University, pl.

M. Curie-Skłodowskiej 3, 20-031 Lublin, Poland

e-mail: mmacieje@umcs.pl synthesized pure and filled with inorganic fillers porous copolymers of 1-vinyl-2-pyrrolidone and divinylbenzene (VP-DVB) [24]. It was found that both textural and thermal properties of the filled materials were quite different comparing with the pure copolymer. Especially significant differences were visible in the case of MCM-41-filler.

Surprisingly, the addition of inorganic filler decreased the thermal resistance of VP-DVB porous copolymer. What is more, the phase separation occurs earlier in the system with the filler, and consequently the value of porous surface area is much lower than in the case of pure copolymers.

Therefore, it was of interest to investigate the influence of inorganic filler on the properties of different types of polymer matrix. Since trimethylolpropane trimethacrylate (TRIM) was earlier applied as crosslinker in synthesis of porous VP-TRIM, this system was chosen for the present investigations. As fillers the same substances were applied as in the previous work: high disperse fumed silica ( $\mathrm{Si}$ ) with the methylsilyl groups in the surface layer, MCM-41 $\mathrm{Si}$, carbon black (C). The thermal properties of the nonfilled and modified copolymers were studied by the means of thermogravimetry (TG) and differential scanning calorimetry (DSC). Additionally the textural characterization was carried out on the basis of the low-temperature nitrogen adsorption on the studied copolymers.

\section{Experimental}

Preparation of copolymers

\section{Chemicals}

TRIM, Merck (Darmstadt, Germany), 1-vinyl-2-pyrrolidone (VP) Fluka (Buchs, Switzerland) were washed with 
$5 \%$ aqueous sodium hydroxide in order to remove inhibitors. Poly(vinyl alcohol) and $\alpha, \alpha^{\prime}$-azoisobisbutyronitrile from Fluka (Buchs, Switzerland) were used without purification. Toluene, $n$-dodecane, acetone, and methanol (reagent grade) were from POCh (Gliwice, Poland).

Preparation of porous microspheres All porous microspheres were obtained by suspension polymerization using equivalent mole fraction of monomers. For copolymerization with VP TRIM as cross-linking agent was used. The process of copolymerization proceeded in the following way: $195 \mathrm{~mL}$ of distilled water and $6.5 \mathrm{~g}$ of poly(vinyl alcohol) were stirred for $6 \mathrm{~h}$ at $80{ }^{\circ} \mathrm{C}$ in the three-necked flask fitted with a stirrer, water condenser, and thermometer. Then the solution containing $15 \mathrm{~g}$ of monomers and $0.075 \mathrm{~g}$ of $\alpha, \alpha^{\prime}$-azoisobisbutyronitrile in $22.5 \mathrm{~mL}$ of toluene was prepared. Next $1.5 \mathrm{~g}$ of filler (high disperse fumed $\mathrm{Si}, \mathrm{MCM}-41$ or C) was added (samples VP-TRIM/Si, VP-TRIM/MCM and VP-TRIM/C, respectively). The polymerization mixture was added while stirring to the aqueous medium. Copolymerization was performed for $20 \mathrm{~h}$ at $80{ }^{\circ} \mathrm{C}$. Porous beads (the diameter range $50-250 \mu \mathrm{m})$ formed in this process were filtered off, washed with hot water, and extracted in a Soxhlet apparatus with acetone, toluene, and methanol. The purified beads were separated into fractions by the sieving.

Methods of analysis Textural characterization of the copolymers was carried out by the low-temperature nitrogen adsorption-desorption method. Nitrogen adsorptiondesorption isoterms were obtained at the liquid nitrogen temperature using a volumetric adsorption analyzer ASAP 2405 (Micromeritics Inc., USA). The measurements of the porous structure of the copolymers were preceded by outgassing of the samples at $140{ }^{\circ} \mathrm{C}$ for $2 \mathrm{~h}$. The specific surface area of the investigated samples was calculated by the Brunauer-Emmet-Teller (BET) method for the adsorption data in the range of a relative pressure $p / p_{\mathrm{o}}$ $0.05-0.25$. The total pore volume was estimated from a single-point adsorption at a relative pressure of 0.985 . The pore size distributions (PSD) were obtained from the desorption branch of the isotherm using the Barrett-JoynerHalenda (BJH) procedure [25].

The maximum of PSD was defined as pore diameter in contrast to the average pore diameter calculated as $D_{\mathrm{p}}=4 V_{\mathrm{p}} S_{\mathrm{BET}}^{-1}$ (on assumption of a cylindrical shape of pores).

Swellability coefficients $(B)$ were determined by equilibrium swelling in acetone, toluene, tetrahydrofuran, and methanol using the centrifugation method. $B$ is expressed as [26]

$B=\frac{V_{\mathrm{s}}-V_{\mathrm{d}}}{V_{\mathrm{d}}} \times 100 \%$ where $V_{\mathrm{s}}$ is volume of the swollen microspheres $(\mathrm{mL}), V_{\mathrm{d}}$ is volume of dry microspheres $(\mathrm{mL})$.

Attenuated total reflection (ATR) was recorded using infrared Fourier transform spectroscopy on spectrometer TENSOR 27 produced by Brucker, Germany, equipped with diamond crystal. The spectra were recorded in the spectral range of $600-4,000 \mathrm{~cm}^{-1}$ with 16 scans per spectrum at a resolution of $4 \mathrm{~cm}^{-1}$.

Elemental analysis of the obtained microspheres was carried out using the Perkin Elmer CHN 2400 apparatus.

The surface of the obtained beads was also examined using an atomic force microscope (AFM), AFM Nanoscope III (Digital Instruments, USA) operating in contact mode. Additionally, the microspheres were imaged using a LEO 1430 VP numerical scanning electron microscope (Germany) with a countershaft and an energy dispersive X-ray detector.

The thermal properties of the synthesized composites were evaluated on the basis of TG and DSC measurements performed using the STA449, F1 Jupiter analyzer from Netzsch (Günzbung, Germany). The procedure was as follows: about $10 \mathrm{mg}$ of the sample was placed in the TG pan and heated in helium or in air atmosphere at a rate of $10 \mathrm{~K} \mathrm{~min}^{-1}$ up to $1,000{ }^{\circ} \mathrm{C}$ with the sample weight about $10 \mathrm{mg}$. The initial decomposition temperature (IDT), $T_{20} \%, T_{50} \%$ of mass loss, and final decomposition temperature (FDT) were determined.

\section{Results and discussion}

Porous copolymers of VP-TRIM used in this study were obtained by suspension copolymerization in the form of regular microspheres (Figs. 1, 2). During the synthesis, three different fillers high disperse fumed Si, MCM-41, and $\mathrm{C}$ were incorporated into polymer network. Table 1 presents the results of elemental analysis of the copolymers under study. Apart from elements listed in the table, the VP-TRIM and VP-TRIM/C copolymers contain $27 \%$ of oxygen whereas VP-TRIM/Si and VP-TRIM/MCM oxygen and silicon. Very important information can be drown on the basis of the nitrogen content in the copolymers. As the nitrogen occurs only in the functional monomer, it was possible to evaluate the molar ratio of VP to crosslinker. The obtained data indicate that comparing with starting polymerization mixture lower amount of VP was incorporated in the copolymer networks. In the initial system, the ratio was $1: 1$; in the obtained products, it fluctuated from 1:0.43 to 1:0.7 depending on the used filler.

Inorganic fillers in the polymerization mixture significantly influence the process of polymerization. 
Table 2 presents the degree of double bounds conversion and main parameters of porous structure of the copolymers under study. Conversion of double bonds in the microspheres synthesized with the use of multifunctional crosslinkers is rarely complete. In order to find the conversion

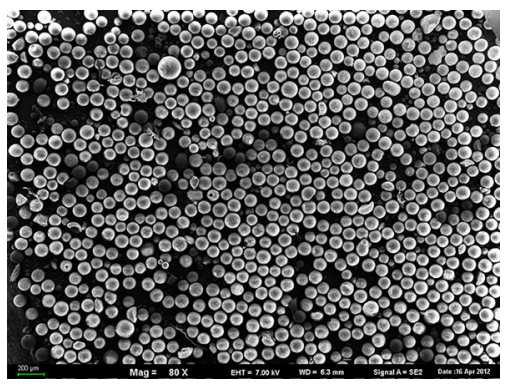

Fig. 1 SEM microphotograph of VP-TRIM material

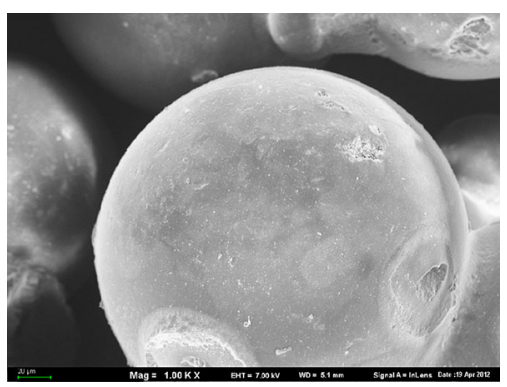

Fig. 2 SEM microphotograph of VP-TRIM/C material

Table 1 Elemental analysis of the copolymers

\begin{tabular}{lllll}
\hline Copolymer & \multicolumn{2}{l}{ mass\% } & & $\begin{array}{l}\text { Mole ratio of VP to TRIM } \\
\text { in the copolymer }\end{array}$ \\
\cline { 2 - 4 } & $\mathrm{C}$ & $\mathrm{H}$ & $\mathrm{N}$ & \\
\hline VP-TRIM & 63.12 & 7.78 & 1.35 & $0.43: 1$ \\
VP-TRIM/C & 62.82 & 7.87 & 2.19 & $0.7: 1$ \\
VP-TRIM/Si & 59.14 & 7.17 & 1.5 & $0.48: 1$ \\
VP-TRIM/MCM & 50.81 & 7.02 & 1.4 & $0.45: 1$ \\
\hline
\end{tabular}

degree, the infrared spectroscopy was applied. Intensities of peaks responsible for stretching vibrations of the $\mathrm{C}=\mathrm{C}$ $\left(1,637 \mathrm{~cm}^{-1}\right)$ group before and after polymerization were compared. As an internal standard, the peak responsible for stretching vibration of carbonyl group $\left(1,720 \mathrm{~cm}^{-1}\right)$ was used. The degree of conversion (DC) was calculated by the following equation:

$D C=100 \%-\left(\frac{\left(\frac{I_{\mathrm{C}=\mathrm{C}}}{I_{\mathrm{C}=0}}\right)_{\text {polymer }}}{\left(\frac{I_{\mathrm{C}=\mathrm{C}}}{I_{\mathrm{C}=0}}\right)_{\text {monomer }}} \times 100 \%\right)$

The obtained values of double bond conversion are summarized in Table 2. Thus, one can assume that presence of the fillers significantly diminishes the conversion of double bounds. It is especially visible in the case of C. The conversion of double bounds in the material obtained in the presence of this filler is almost $20 \%$ lower than in the pure VP-TRIM copolymer.

Investigation of porous structure of the copolymers displayed that VP-TRIM copolymer possesses the highest value of specific surface area $\left(607 \mathrm{~m}^{2} \mathrm{~g}^{-1}\right)$ and pore volume $\left(1.15 \mathrm{~cm}^{3} \mathrm{~g}^{-1}\right)$. Addition of the fillers diminishes these parameters. It is especially visible in the case of VPTRIM/MCM copolymer. Despite the fact that MCM-41 has itself well developed porous structure, the specific surface area of VP-TRIM/MCM composite is much lower (224 $\mathrm{m}^{2} \mathrm{~g}^{-1}$ comparing with $1,012 \mathrm{~m}^{2} \mathrm{~g}^{-1}$ of pure MCM41 and $607 \mathrm{~m}^{2} \mathrm{~g}^{-1}$ of VP-TRIM copolymer). Two other fillers also influence the formation of porous structure. This effect could be explained by the fact that during synthesis the phase separation occurs very early in the system with the filler. Therefore, the nuclei of polymers in formation could capture preferably monomers from the local solution, and consequently increase their size and the sizes of pores formed between them. As a consequence, the value of porous structure is quite low, whereas the pore volume is considerable.

The differences in internal structures created during the polymerization with various fillers can be also observed using AFM. AFM images of the copolymers with different

Table 2 Degrees of double bond conversion and parameters of the porous structure of the materials under study

\begin{tabular}{|c|c|c|c|c|}
\hline Material & $\begin{array}{l}\text { Degrees of double } \\
\text { bond conversion }\end{array}$ & $\begin{array}{l}\text { Specific surface } \\
\text { area } S_{\mathrm{BET}} / \mathrm{m}^{2} \mathrm{~g}^{-1}\end{array}$ & $\begin{array}{l}\text { Pore volume } \\
V / \mathrm{cm}^{3} \mathrm{~g}^{-1}\end{array}$ & $\begin{array}{l}\text { Pore diameter } \\
D_{\mathrm{BJH}} / \mathrm{nm}\end{array}$ \\
\hline Fumed silica ( $\mathrm{Si}$ ) & - & 330 & 0.64 & 9.5 \\
\hline MCM-41 & - & 1012 & 1.16 & 4.0 \\
\hline Carbon black (C) & - & 386 & 0.28 & 4.9 \\
\hline VP-TRIM & 87 & 607 & 1.15 & 11.0 \\
\hline VP-TRIM/C & 68 & 307 & 0.92 & 10.2 \\
\hline VP-TRIM/Si & 81 & 467 & 0.97 & 7.8 \\
\hline VP-TRIM/MCM & 84 & 224 & 0.56 & 9.7 \\
\hline
\end{tabular}


(a)

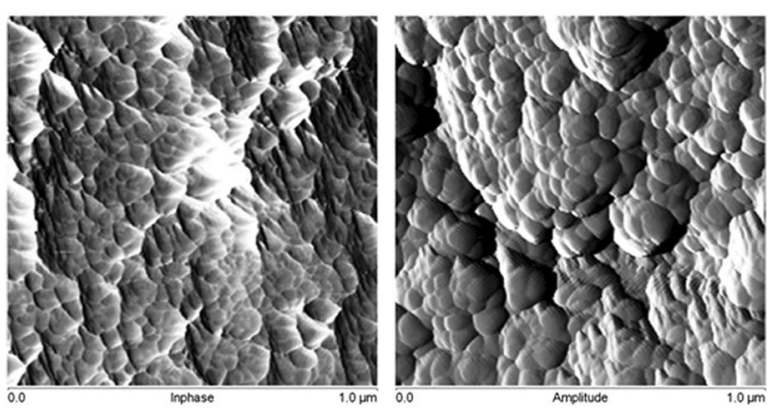

(b)

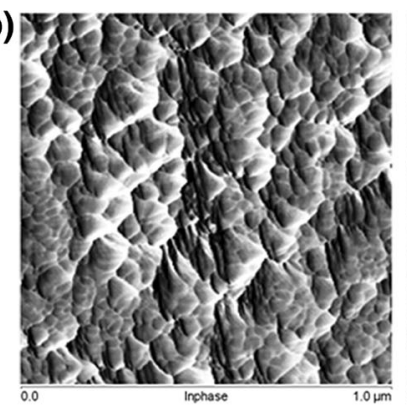

(c)

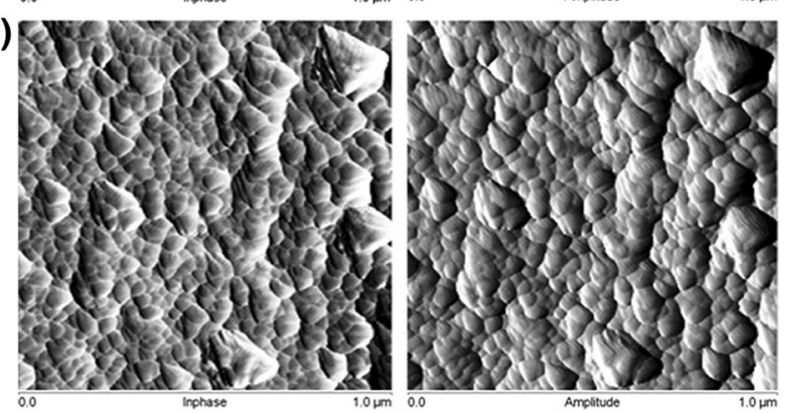

Fig. 3 AFM image of the copolymers synthesized with different fillers a VP-TRIM/MCM; b VP-TRIM/Si; $\mathbf{c}$ VP-TRIM/C

fillers are presented in Fig. 3. On the basis of AFM measurements, the root mean squares (RMSs) roughness for the studied copolymers was calculated. The RMSs are as follows: VP-TRIM/MCM-36 nm, VP-TRIM/Si-48 nm, and VP-TRIM/C-42 nm. Hence, one can observe that they are directly proportional to the values of surface area of the samples under study.

Table 3 presents the swellability coefficients of copolymers in acetone, methanol, toluene, and tetrahydrofuran. As the coefficients depend on the porous structure, chemical composition and double bounds conversion there is not simple correlation between the ability to swell and used filler.

The main goal of this work was to investigate the influence of the fillers on the thermal properties of the discussed materials. Table 4 presents the IDT, $T_{50} \%$ of mass loss, and FDT in helium. From these data, one can see that addition of inorganic fillers lowers the IDT of the copolymers. This effect is especially visible in the case of VP-TRIM/C
Table 3 Swellability coefficients of copolymers under study

\begin{tabular}{lllll}
\hline Copolymer & \multicolumn{4}{l}{ Swellability coefficients $B / \%$} \\
\cline { 2 - 5 } & Acetone & Methanol & Toluene & Terahydrofuran \\
\hline VP-TRIM & 36 & 20 & 40 & 80 \\
VP-TRIM/C & 40 & 20 & 20 & 30 \\
VP-TRIM/Si & 38 & 38 & 40 & 40 \\
VP-TRIM/MCM & 40 & 45 & 35 & 40 \\
\hline
\end{tabular}

Table 4 Thermal stability of the copolymers under study determined in helium

\begin{tabular}{llllll}
\hline Copolymer & $\mathrm{IDT} /{ }^{\circ} \mathrm{C}$ & $T_{20} \% /{ }^{\circ} \mathrm{C}$ & $T_{50 \%} /{ }^{\circ} \mathrm{C}$ & $\mathrm{FDT} /{ }^{\circ} \mathrm{C}$ & $T_{\max }$ \\
\hline VP-TRIM & 341 & 386 & 438 & 503 & 447 \\
VP-TRIM/C & 228 & 376 & 435 & 499 & 458 \\
VP-TRIM/Si & 307 & 348 & 397 & 650 & 376 \\
VP-TRIM/MCM & 310 & 353 & 405 & 504 & 410 \\
\hline
\end{tabular}

material. Its initial temperature is more than $100{ }^{\circ} \mathrm{C}$ lower than in the case of pure VP-TRIM copolymer. In the case of VP-DVB/Si and VP-DVB/C materials, their IDTs are also lower but the differences are about $30{ }^{\circ} \mathrm{C}$. The temperature of the maximum of mass loss of pure VP-TRIM copolymer is $503{ }^{\circ} \mathrm{C}$. The addition of MCM and $\mathrm{C}$ to the copolymers has not changed significantly the position of the FDT. The situation was different when fumed $\mathrm{Si}$ was used as a filler. In this case, thermal degradation of the material proceeds through two stages. The first step at $450{ }^{\circ} \mathrm{C}$ is followed by second stage above $650{ }^{\circ} \mathrm{C}$. Graphical representation of the thermal behaviour of the discussed material in helium atmosphere is presented in Fig. 4.

Figure 5 presents the thermal degradation of the copolymers under study in air. What is interesting the IDT for VP-TRIM, VP-TRIM/Si, and VP-TRIM/MCM are lower than in helium whereas for VP-TRIM/C is about $30{ }^{\circ} \mathrm{C}$ higher (Table 5).

Generally, the carbon filler has the most significant impact on the thermal properties of the obtained materials. It is directly connected with the double bonds conversion. For VP-TRIM/C material, it has the lowest value and consequently the thermal resistance of this copolymer is the poorest among all of the discussed materials. For VP-DVB series, similar effect was observed for the MCM (the most polar filler). Application of MCM in the synthesis of VPDVB copolymers resulted in huge drop of the IDT. As TRIM has more polar character than DVB in the synthesis of VP- 
TRIM series, the most spectacular differences are visible in the case of the less polar filler $(\mathrm{C})$.

The DSC curves of copolymers under study are presented in Fig. 6. DSC analysis showed similarity in thermal behaviour of all prepared copolymers. They had a characteristic, well-shaped calorimetric profile. The first, endothermic peak $\left(T_{\mathrm{d} 1}\right)$ can be attributed to the desorption of water that is present into internal structure of porous copolymers. The addition of the fillers has not changed significantly the position of the decomposition peaks $\left(T_{\mathrm{d} 2}\right)$. The only change is that enthalpy of decomposition $\left(\Delta H_{\mathrm{d} 2}\right)$ has lower value due to the presence of the fillers (Table 6).

To complete the study, AFM based mechanical mapping techniques were applied to determine the Young's modulus. The morphologies of the samples under study are visualized in Fig. 7. As can be seen, differences in the DC and the chemical composition of the obtained materials are also reflected in the value of Young's modulus (Table 7). Addition of $\mathrm{C}$ diminishes Young's modulus value. This phenomenom can be attributed to the relatively low conversion of double bonds. In the case of MCM and Si fillers, the values of Young's moduli are higher comparing with pure VP-TRIM copolymer.

Table 5 Thermal stability of the copolymers under study determined in air

\begin{tabular}{llllll}
\hline Copolymer & $\mathrm{IDT} /{ }^{\circ} \mathrm{C}$ & $T_{20} \% /{ }^{\circ} \mathrm{C}$ & $T_{50 \%} /{ }^{\circ} \mathrm{C}$ & $\mathrm{FDT} /{ }^{\circ} \mathrm{C}$ & $T_{\max }$ \\
\hline VP-TRIM & 282 & 320 & 352 & 620 & 338 \\
VP-TRIM/C & 260 & 318 & 368 & 615 & 330 \\
VP-TRIM/Si & 288 & 319 & 364 & 640 & 324 \\
VP-TRIM/MCM & 283 & 314 & 344 & 620 & 322 \\
\hline
\end{tabular}

Fig. 4 The TG curves of VP-TRIM/Si (1), VP-TRIM/ MCM (2), VP-TRIM/Si (3), VP-TRIM (4) determined in helium

Fig. 5 The TG curves of VP-TRIM (1), VP-TRIM/C (2), VP-TRIM/MCM (3), VP-TRIM/Si (4) determined in air
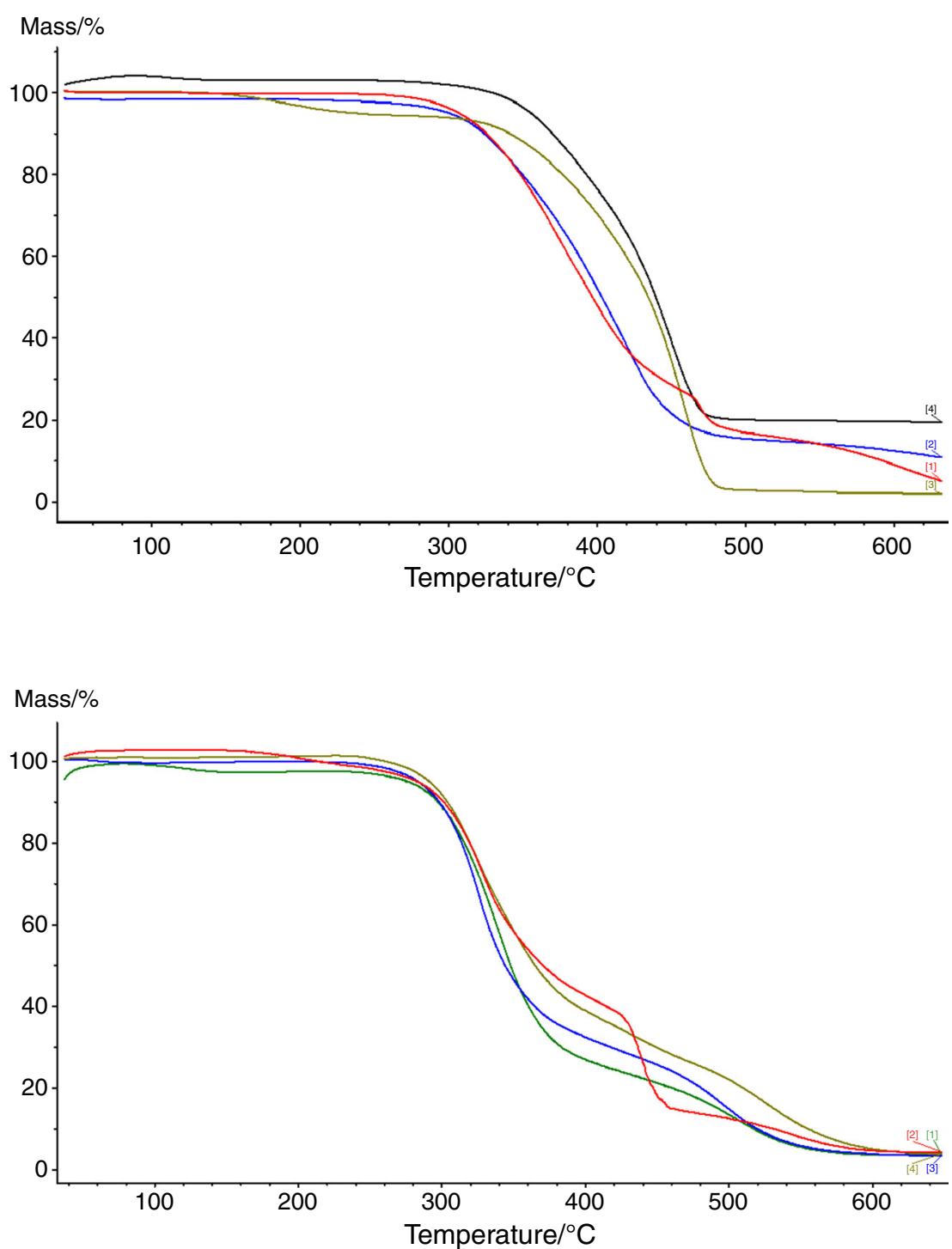
Fig. 6 Comparison of the DSC curves of the copolymers under study [1] VP-TRIM,

[2] VP-TRIM/C, [3] VP-TRIM/ MCM, [4] VP-TRIM/Si

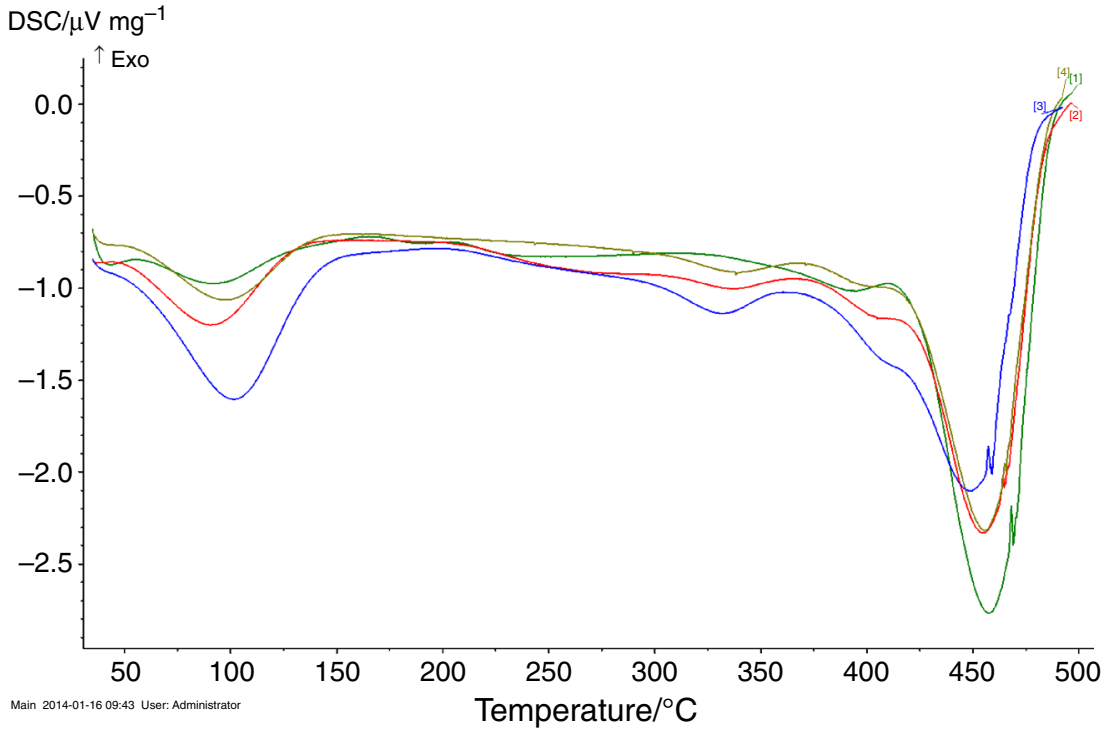

Table 6 DSC data determined for the copolymers under study

\begin{tabular}{llcll}
\hline Copolymer & $\begin{array}{l}T_{\mathrm{d} 1} / \\
{ }^{\circ} \mathrm{C}\end{array}$ & $\begin{array}{l}\Delta H_{\mathrm{d} 1} / \\
\mu \mathrm{V} \mathrm{mg}\end{array}$ & $\begin{array}{l}T_{\mathrm{d} 2} / \\
{ }^{\circ} \mathrm{C}\end{array}$ & $\begin{array}{l}\Delta H_{\mathrm{d} 2} / \\
\mu \mathrm{V} \mathrm{mg}\end{array}$ \\
\hline VP-TRIM & 92 & 61 & 457 & 760 \\
VP-TRIM/C & 90.3 & 107 & 455 & 560 \\
VP-TRIM/Si & 97 & 96 & 455 & 443 \\
VP-TRIM/MCM & 101.5 & 207 & 448 & 437 \\
\hline
\end{tabular}
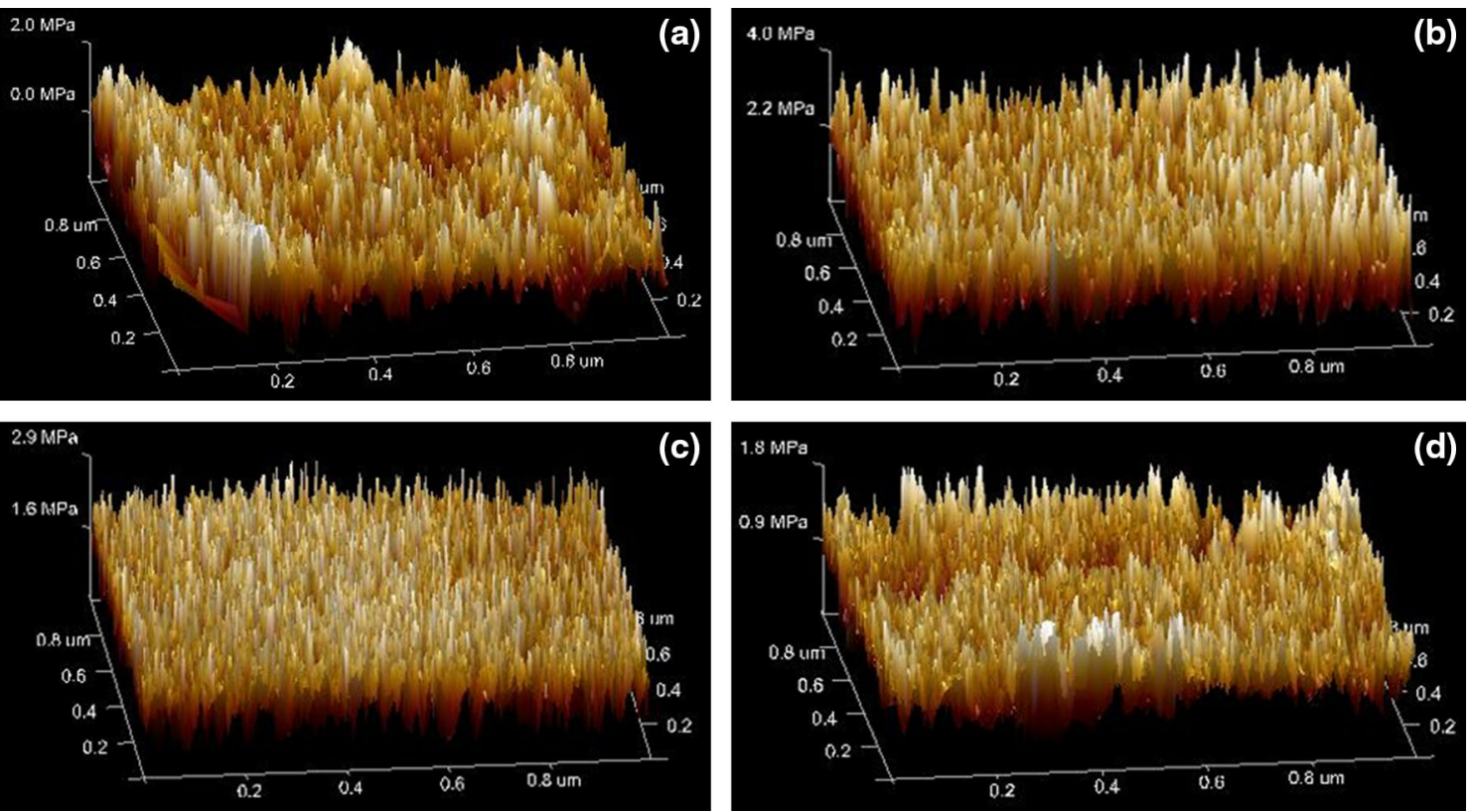

Fig. 7 Visualization of the Young's moduli determined on the basis of AFM measurements VP-TRIM (a), VP-TRIM/MCM (b), VP-TRIM/Si (c), VP-TRIM/C (d) 


\section{Conclusions}

The synthesis and characterization of textural and thermal properties of non-filled and filled with inorganic fillers porous copolymers of VP-TRIM were presented. It was found that during the synthesis in the presence of inorganic filler the phase separation occurs earlier in the system and consequently the value of porous surface area is much lower than in the case of pure copolymers. Especially significant effect on the porous structure has MCM-41-filler. On the other hand, C filler is responsible for the low conversion of double bonds. It results in diminishing both thermal and mechanical properties of the copolymers obtained in the presence of this filler. The obtained results are analogical ones in VP-DVB system and indicate that in the case of porous copolymers the presence of inorganic fillers results in deterioration of thermal and mechanical properties of the obtained materials.

Acknowledgements The project was supported the European Community's Seventh Framework Programme (FP7/2007-2013) under a Marie Curie International Research Staff Exchange Scheme, Grant Agreement No PIRSES-GA-2008-230790.

Open Access This article is distributed under the terms of the Creative Commons Attribution License which permits any use, distribution, and reproduction in any medium, provided the original author(s) and the source are credited.

\section{References}

1. Apopei DF, Dinu MV, Trochimczuk AW, Dragan ES. Sorption isotherms of heavy metal ions onto semi-interpenetrating polymer network cryogels based on polyacrylamide and anionically modified potato starch. Ind Eng Chem Res. 2012;51:10462-71.

2. Drechny D, Trochimczuk AW. Synthesis and some sorptive properties of highly crosslinked cyanomethyl styrene/divinylbenzene copolymers. React Funct Polym. 2006;66:323-33.

3. Trochimczuk AW, Streat M, Kolarz N. Highly polar polymeric sorbents: characterization and sorptive properties towards phenol and its derivatives. React Funct Polym. 2001;46:259-71.

4. Jaćkowska M, Bocian S, Gawdzik B, Grochowicz M, Buszewski B. Influence of chemical modification on the porous structure of polymeric adsorbents. Mater Chem Phys. 2011;130:644-50.

5. Maciejewska M, Gawdzik J. Preparation and characterization of sorption properties of porous microspheres of 1-vinyl-2-pyrrolidone-divinylbenzene. J Liq Chromatogr Relat Technol. 2008;31:950-61.

6. Bielicka-Daszkiewicz K, Voelkel A, Szejner M, Osypiuk J. Extraction properties of new polymeric sorbents in SPE/GC analysis of phenol and hydroquinone from water samples. Chemosphere. 2006;62:890-8.
7. Maciejewska M. Characterization of macroporous 1-vinyl-2pyrrolidone copolymers obtained by suspension polymerization. J Appl Polym Sci. 2012;124:568-75.

8. Maciejewska M, Osypiuk-Tomasik J. Studies of sorption properties of porous copolymers of 1-vinyl-2-pyrrolidone. J Therm Anal Calorim. 2013;111:1595-601.

9. Grochowicz M, Gawdzik B. Preparation and characterization of porous crosslinked microspheres of new aromatic methacrylates. J Porous Mater. 2013;20:339-49.

10. Maciejewska M, Szajnecki $Ł$, Gawdzik B. Investigation of the surface area and polarity of porous copolymers of maleic anhydride and divinylbenzene. J Appl Polym Sci. 2012;125:300-7.

11. Gokmen MT, Du Prez FE. Porous polymer particles-a comprehensive guide to synthesis, characterization, functionalization and applications. Prog Polym Sci. 2012;37:365-405.

12. Maciejewska M, Osypiuk-Tomasik J. Sorption on porous copolymers of 1-vinyl-2-pyrrolidone-divinylbenzene. J Therm Anal Calorim. 2013;114:749-55.

13. Podkościelna B, Maciejewska M, Bartnicki A. Studies on synthesis and physicochemical properties of new bis[4-(2-hydroxy-3methacryloyloxypropoxy) phenyl]sulfide terpolymers. J Appl Polym Sci. 2012;123:59-65.

14. Gawdzik B, Maciejewska M. Synthesis of isobutylmaleate-divinylbenzene microspheres by different techniques of heterogeneous polymerizations. J Appl Polym Sci. 2004;91:2008-15.

15. Bolbukh YN, Tertykh VA, Gawdzik B. TG and DSC studies of filled porous copolymers. J Therm Anal Calorim. 2006;86: 125-32.

16. Zaleski R, Stefaniak W, Maciejewska M, Goworek J. Porosity evolution of VP/DVB/MCM-41 nanocomposite. J Colloid Interface Sci. 2010;343:134-40.

17. Zaleski R, Kierys A, Grochowicz M, Dziadosz M, Goworek J. Synthesis and characterization of nanostructural polymer-silica composite: positron annihilation lifetime spectroscopy study. J Colloid Interface Sci. 2011;358:268-76.

18. Kierys A, Zaleski R, Grochowicz M, Goworek J. Thinning down of polymer matrix by entrapping silica nanoparticles. Colloid Polym Sci. 2011;289:751-8.

19. Leblanc JL. Rubber-filler interaction and rheological properties in filled compounds. Prog Polym Sci. 2002;27:627-87.

20. Katz HS, Mileski JV. Handbook of Fillers for Plastics. New York: Van Nostrand Reinhold; 1987.

21. Alvarez VA, Perez CJ. Effect of different inorganic filler over isothermal and non-isotermal crystallization of polypropylene homopolymer. J Therm Anal Calorim. 2012;107:633-43.

22. Lubin G. Handbook of Composities. London: Chapman \& Hall; 1998

23. Bolbukh Yu, Tertykh V, Klonos P, Pissis P. DSC study of polyhydroxyethylmethacrylate filled with modified silicas. J Therm Anal Calorim. 2012;108:1111-9.

24. Maciejewska M, Osypiuk-Tomasik J. TG/DSC studies of modified 1-vinyl-2-pyrrolidone-divinylbenzene copolymers. J Therm Anal Calorim. 2013;113:343-50.

25. Gregg SJ, Sing KSW. Adsorption, Surface Area and Porosity. London: Academic Press; 1982.

26. Tuncel A, Ecevit K, Kesenci K, Piskin E. Nonswellable and swellable ethylene glycol dimethacrylate-acrylic acid copolymer microspheres. J Polym Sci A. 1996;34(1):45-55. 